\title{
Basal body structure in Trichonympha
}

\author{
Paul Guichard ${ }^{1 *}$ and Pierre Gönczy ${ }^{2^{*}}$
}

\begin{abstract}
Trichonympha is a symbiotic flagellate of many species of termites and of the wood-feeding cockroach. Remarkably, this unicellular organism harbors up to over ten thousand flagella on its surface, which serve to propel it through the viscous environment of the host hindgut. In the 1960s, analysis of resin-embedded Trichonympha samples by electron microscopy revealed that the basal bodies that give rise to these flagella are exceptionally long, with a proximal, cartwheel-bearing, region some 50 times longer than that of regular centrioles. In recent years, this salient feature has prompted the analysis of the 3D architecture of Trichonympha basal bodies in the native state using cryo-electron tomography. The resulting $\sim 40 \AA$ resolution map of the basal body proximal region revealed a number of novel features that may be conserved in centrioles of other systems. These include proximal-distal polarity of the pinhead structure that links the cartwheel to centriolar microtubules, as well as of the linker between the $\mathrm{A}$ and the $\mathrm{C}$ microtubules. Moreover, this work demonstrated that the cartwheel is made of stacked ring-like structures that likely each comprise 18 molecules of SAS-6 proteins.
\end{abstract}

Keywords: Trichonympha, Long centriole, Cartwheel, Cryo-tomography

\section{Organism}

Trichonympha sp. is an anaerobic symbiotic flagellate, which is part of the parabasalid goup, within the supergroup Excavata [1]. Parabasalids are characterized by parabasal fibers that link their basal bodies to Golgi complexes. Trichonympha sp. cells are typically $\sim 100 \mu \mathrm{m}$ long and lack mitochondria [2]. Note that, unless otherwise stated, the descriptions below pertain to several Trichonympha species that share essentially the same basic structural features. The same holds for the related Pseudotrichonympha sp., as well as the two Australian species Deltotrichonympha operculata and Koruga bonita [3-6].

\section{Basic basal body structure and composition}

Canonical microtubule triplets are present in Trichonympha basal bodies. Over ten thousand flagella that stem from basal bodies have been estimated to be present on a single Trichonympha campanula cell [5, 7] (Fig. 1). In addition, two centrioles are present close to the rostrum

\footnotetext{
*Correspondence: paul.guichard@unige.ch; pierre.gonczy@epfl.ch

${ }^{1}$ Department of Cell Biology, University of Geneva, Geneva, Switzerland

2 Swiss Institute for Experimental Cancer Research (ISREC), School

of Life Sciences, Swiss Federal Institute of Technology (EPFL), Lausanne, Switzerland
}

(Fig. 1), one long one and one short one [6]. Both delta tubulin and epsilon tubulin are present in the genome of Trichonympha agilis [8], although whether the corresponding proteins localize to the basal body is not known.

\section{Additional basal body structures or accessory structures}

A salient characteristic of Trichonympha basal bodies is their unusual length, which results from an extended proximal region (Fig. $2 \mathrm{a}-\mathrm{c}$ ) that bears the cartwheel (Fig. 2d), and which can be up to $5 \mu \mathrm{m}$ long, instead of the usual $100 \mathrm{~nm}[5,8]$. Basal body length varies slightly depending on the position in the cell, with basal bodies in the anteriorly located rostrum being shorter than those in the cell posterior [6]. By contrast to the unusually long proximal region, the distal region of Trichonympha basal bodies resembles that of other centrioles, including in terms of length, as well as the presence of doublet microtubules and of centrin (Fig. 2a-c) [8, 9]. Distal appendages appear to be present as well, whereas no clear sub-distal appendages have been observed [5]. The first comprehensive ultrastructural analysis of basal bodies in Trichonympha using electron microscopy is to be credited to Gibbons and Grimstone [5]. 


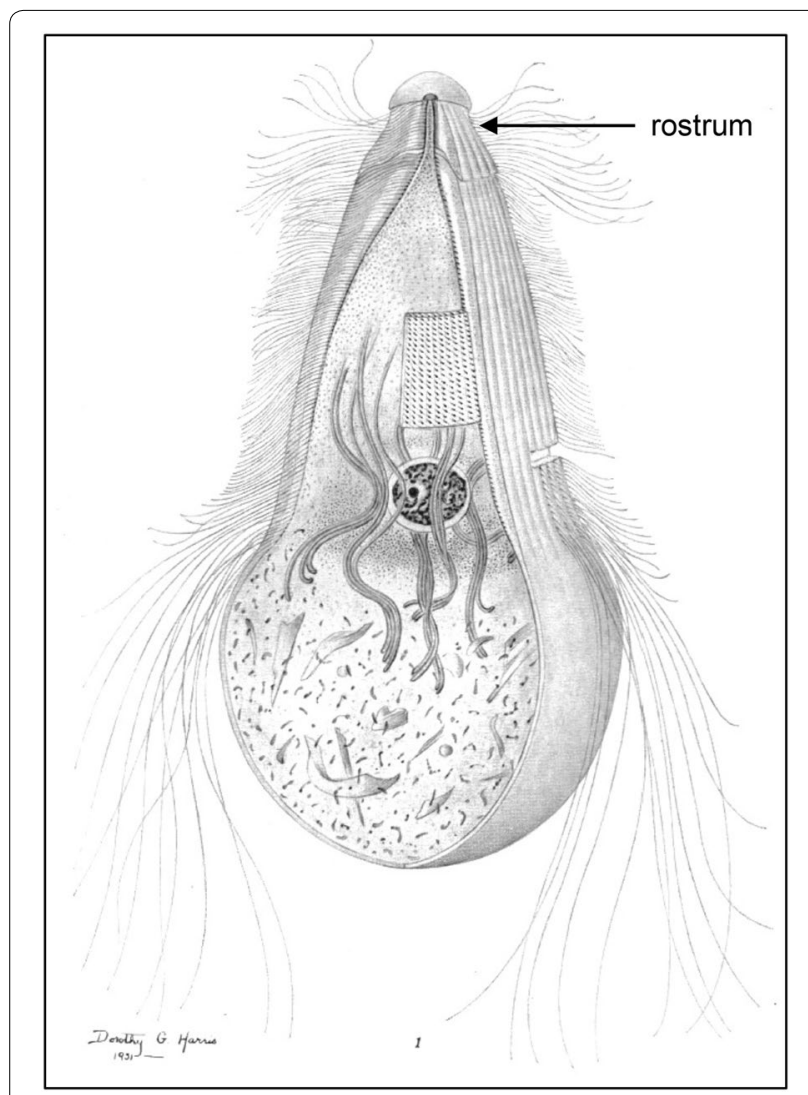

Fig. 1 Trichonympha collaris. The entire Trichonympha cell is covered by flagella, except for the bottom part, where pieces of wood can be seen inside the cell. The rostrum is located at the top of the cell as indicated. A Trichonympha collaris cell is approximately $100 \mu \mathrm{m}$. Image from Kirby [7]

\section{Basal body origins}

How basal bodies form in Trichonympha is not known with certainty. In Deltotrichonympha operculata, basal bodies are thought to form from short subunits called kinetosomes that are arranged in a columnar fashion in the anterior region of the cell $[3,4]$. It is not clear whether basal bodies form de novo or in the vicinity of existing ones.

\section{Basal body life cycle and other functions}

The basal body life cycle has been investigated in Trichonympha magna [10], where the long and short centrioles located close to the rostrum seem to act as centrosomes during mitosis, while the basal bodies remain at the cell surface during cell division.

\section{Identification of basal body components}

No proteomic or genomic screen has been performed to date. However, the genome of Trichonympha agilis is currently being assembled, which already allowed the identification of mRNAs encoding TaSAS-6 (Fig. 2b), $\alpha$-tubulin, $\beta$-tubulin, $\delta$-tubulin, $\varepsilon$-tubulin [8], as well as centrin (PaG and PiG, unpublished observations).

\section{Notable basal body findings}

The 3D architecture of the cartwheel-bearing region of basal bodies in their native state has been elucidated using cryo-microscopy coupled to tomography (Fig. 3ac) [8]. The resulting $\sim 40 \AA$ resolution map revealed notably the existence of proximal-distal polarity both in the pinhead that links the cartwheel to centriolar microtubules, as well as in the linker situated between the $\mathrm{A}$ and the $\mathrm{C}$ microtubules (Fig. 3c). Moreover, this analysis demonstrated that the cartwheel comprises a stack of ring-like structures that are $\sim 4.5 \mathrm{~nm}$ high and which exhibit a periodicity of $\sim 8.25 \mathrm{~nm}$ in the centriole center; by contrast, the periodicity at the periphery is of $\sim 16.5 \mathrm{~nm}$ as a result from the merger of spokes from two superimposed rings. This work uncovered also that ringlike assemblies of 18 molecules of SAS-6 proteins that were postulated based on structural work to be at the

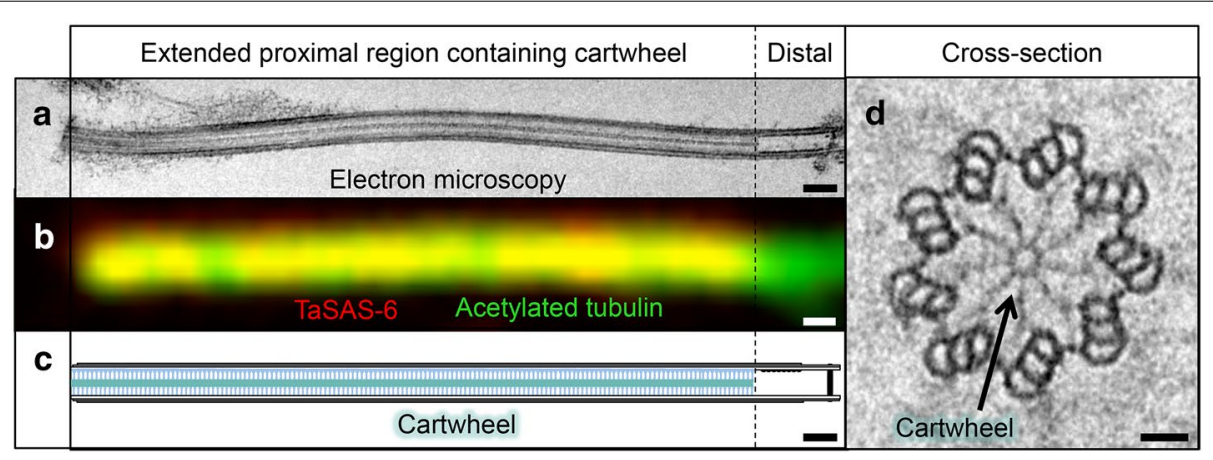

Fig. 2 Exceptionally long basal body in Trichonympha. a Electron micrograph of isolated Trichonympha sp. basal body. b Immunofluorescence of isolated Trichonympha sp. basal body revealing TaSAS-6 localization (red, yellow in overlay) along the basal body/flagellum complex stained with acetylated tubulin (green). c Schematic representation of the exceptionally long basal body of Trichonympha, with the cartwheel-bearing region highlighted. d Cross section of Trichonympha basal body; arrow points to cartwheel structure, with central hub and nice radial spokes connecting with peripheral microtubules. Scale bar in (a, b) $250 \mathrm{~nm}$, in (d) $50 \mathrm{~nm}$ 


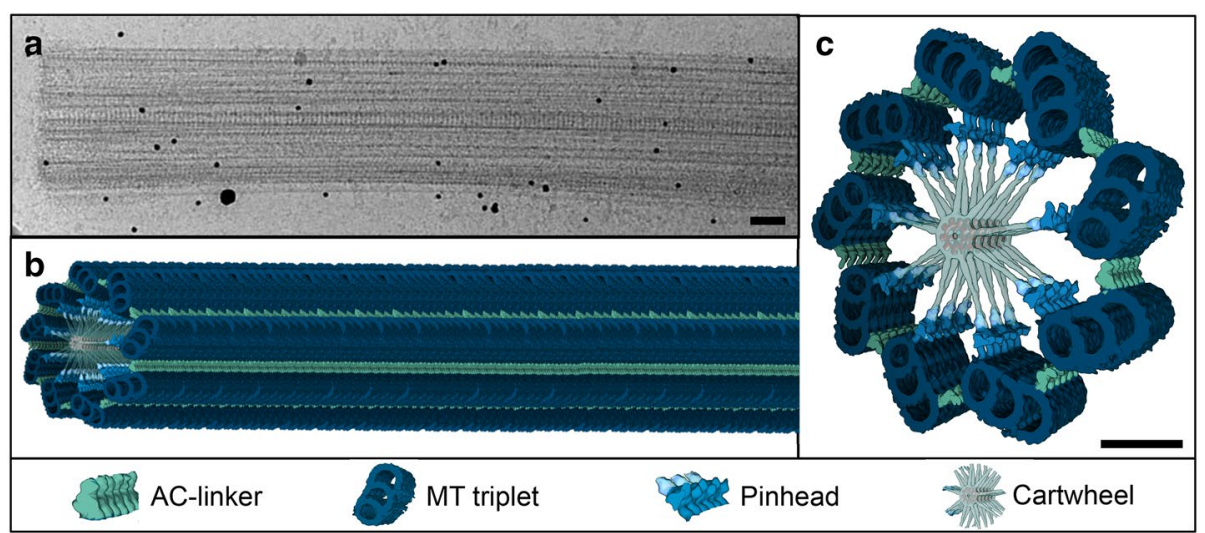

Fig. 3 Native structure of the proximal region of Trichonympha basal body. a Cryo-microscopy image of isolated Trichonympha sp. basal body viewed from the side. Scale bar $100 \mathrm{~nm}$. b-c 3D architecture of the cartwheel-bearing region of basal bodies at $\sim 40 \AA$ resolution, side-view (b) and cross section (c). Scale bar $50 \mathrm{~nm}$

core of the cartwheel fit well in the cryo-EM map of the Trichonympha basal body [8-11].

\section{Strengths and future of basal body research in Trichonympha}

One obvious strength of Trichonympha as a model system is the large number of basal bodies per cell, which can be easily purified together with the flagella. In addition, the exceptionally long proximal region offers remarkable opportunities for structural analysis by cryotomography. However, the anaerobic life cycle and the lack of transgenesis and gene inactivation methods preclude at present functional studies in Trichonympha. In the future, Trichonympha could be used for proteomic analysis of purified basal bodies, in particular, to uncover proteins specific of the proximal region. Furthermore, the $5-\mu \mathrm{m}$-long centriole is a particularly suitable sample for accurate protein localization using super-resolution microscopy.

\section{Abbreviation \\ cryo-EM: cryo-electron microscopy.}

\section{Authors' contributions}

$\mathrm{PaG}$ and $\mathrm{PiG}$ generated the figures and wrote the manuscript. Both authors read and approved the final manuscript.

\section{Acknowledgements}

We are grateful to Virginie Hamel and Veronika Villimova for critical reading of the manuscript. We thank Davide Demurtas (BioEM core facility, EPFL) for the cryo-EM image of Trichonympha basal body. This work was supported by grants from the Swiss National Science Foundation (SNSF) to PaG (PP00P3_157517) and the European Research Council (ERC) to PiG (AdG 233335).

\section{Competing interests}

The authors declare that they have no competing interests.

Received: 27 November 2015 Accepted: 10 February 2016

Published online: 01 March 2016

\section{References}

1. Dack JB, Redfield RJ. Phylogenetic placement of Trichonympha. J Eukaryot Microbiol. 45(4):445-7.

2. Biagini GA, Finlay BJ, Lloyd D. Evolution of the hydrogenosome. FEMS Microbiol Lett. 1997;155(2):133-40.

3. Tamm S, Tamm SL. Origin and development of free kinetosomes in the flagellates Deltotrichonympha and Koruga. J Cell Sci. 1980;42:189-205.

4. Tamm SL. Free kinetosomes in Austrialian flagellates I. Types and spatial arrangement. J Cell Biol. 1972;54(1):39-55.

5. Gibbons IR, Grimstone V. On flagellar structure in certain flagellates. J Biophys Biochem Cytol. 1960;7(4):697-716.

6. Grimstone AV, Gibbons IR. The fine structure of the centriolar apparatus and associated structures in the complex flagellates Trichonympha and Pseudotrichonympha. Philos Trans R Soc B Biol Sci. 1966;250(766):215-42.

7. Kirby H. Flagellates of the genus trichonympha in termites. Univ Calif Publ Zool. 1932;37:349-476.

8. Guichard P, Hachet V, Majubu N, Neves A, Demurtas D, Olieric N, Fluckiger I, Yamada A, Kihara K, Nishida Y, Moriya S, Steinmetz MO, Hongoh Y, Gönczy P. Native architecture of the centriole proximal region reveals features underlying its 9-fold radial symmetry. Curr Biol. 2013;23(17):1620-8.

9. Guichard P, Hamel V, Neves A, Gönczy P. Isolation, cryotomography, and three-dimensional reconstruction of centrioles. Methods Cell Biol. 2015;129:191-209.

10. Cleveland LR. The centrioles of Trichonympha from termites and their functions in reproduction. J Protozool. 1960;7(4):326-41.

11. Guichard P, Desfosses A, Maheshwari A, Hachet V, Dietrich C, Brune A, Ishikawa T, Sachse C, Gönczy P. Cartwheel architecture of Trichonympha basal body. Science. 2012;337(6094):553. 